\title{
Germanica
}

contemporain : jeux intermédiaux, modes de transfert, adaptations

\section{Funktionen ikonischer Zeichen in Romanverfilmungen Das Parfum und Der Vorleser}

Functions of iconic signs in film adaptations The Perfume and The Reader Les fonctions des signes iconiques dans les adaptations cinématographiques Le Parfum et Le Liseur

\section{Brahim Moussa}

\section{(2) OpenEdition}

\section{Journals}

Édition électronique

URL : http://journals.openedition.org/germanica/2269

DOI : 10.4000/germanica.2269

ISSN : 2107-0784

Éditeur

Université de Lille

Édition imprimée

Date de publication : 30 décembre 2013

Pagination : 29-43

ISBN : 9782913857322

ISSN : 0984-2632

Référence électronique

Brahim Moussa, « Funktionen ikonischer Zeichen in Romanverfilmungen Das Parfum und Der Vorleser », Germanica [Online], 53 | 2013, Online erschienen am: 30 Dezember 2016, abgerufen am 06 Oktober 2020. URL : http://journals.openedition.org/germanica/2269 ; DOI : https://doi.org/10.4000/ germanica.2269 


\title{
Funktionen ikonischer Zeichen in Romanverfilmungen Das Parfum und Der Vorleser
}

\author{
Brahim MousSA \\ Université de Carthage
}

Textnarration und Filmnarration erfolgen auf unterschiedliche Art und Weise. Da das Medium im Text - im engsten Sinne des Wortes die Sprache ist, während im Film mehrere Kanäle, wie Kameraführung, Einstellungen, Musik und Ton die Narration bestimmen, lassen sich Vergleiche zwischen Text- und Filmnarration schwer durchführen. Gleichwohl sind zwischen beiden Medien insofern Parallelen zu finden, als die erzählte Welt in einem literarischen Text und die Diegese eines Films vergleichbare Baustrukturen aufweisen können. Denn eine Folge von Ereignissen, die in einem Text das Aufeinanderwirken von Wörtern und Syntagmen begünstigt, lässt sich ebenfalls in einem Film erkennen, wenn auch die Ereignisfolgen hier durch (Bild-)Einstellungen und Sequenzen gebildet werden. Wie im Text entsteht im Film ,eine Art solidarisches Syntagma, innerhalb dessen die ,Einstellungen“" (semantisch) aufeinander wirken" 1 , was auf mögliche Übereinstimmungen mit Textsyntagmen schließt, zumal eine Verkettung von Einstellungen genauso gut einen Mitteilungswert wie sprachliche Syntagmen in einem Text hat. Film und Text sind also beide Erzählmedien. Sie geben Geschichten wieder: „Film und Roman erzählen beide lange

1. - Christian Metz, „Probleme der Denotation im Spielfilm“, in: Franz-Josef Albersmeier (Hrsg.), Texte zur Theorie des Films, Stuttgart, Reclam, 2003, S. 321-370, hier S. 328. 
Geschichten mit einer Fülle von Details [...]. Was immer gedruckt im Roman erzählt werden kann, kann im Film annähernd verbildlicht oder erzählt werden" "2, da eine Geschichte - im plattesten Sinne - das einstimmige Zusammenwirken von Elementen, die nach Gesetzmäßigkeit(en) „auseinander folgen“, d. h. nach einer eruierbaren Motivierung ${ }^{3}$. Was die Verkettung von Erzählsegmenten im Text die Sprache leistet, erfüllt die Bildfolge im Film. Wir können im Film von Geschichte sprechen, „sobald in der Zusammenfügung von zwei oder mehr Bildern etwas zum Ausdruck kommt, was in den einzelnen Bildern nicht enthalten ist" ${ }^{\text {" }}$. Die Montage der Bildeinstellung erzählt im Film die Geschichte.

Kein Wunder also, angesichts dieser Schnittmengen zwischen Romantext und Film, dass sich die Filmforschung Ansätze der Literaturwissenschaft sowie der Erzähltheorie bedient. Jean Marie Peters fasst bereits in den 60er Jahren die Kinobilder als „Filmsprache“ auf: „Was die Struktur und Funktion der gebrauchten Zeichen und Zeichengruppen angeht, besteht also in wesentlichen Punkten eine Übereinstimmung zwischen Wortsprache und Film ${ }^{\text {“5 }}$. Christian Metz ${ }^{6}$ und Peter Wollen ${ }^{7}$ machen in ihren Filmforschungen sowohl von strukturalistischen Theorien als auch von der Peirceschen Zeichenlehre Gebrauch. Markus Kuhn betreibt in seinem Werk Filmnarratologie eine Filmanalyse auf der Basis einer Erzähltheorie à la Gérard Genette. Das sind Beispiele aus einer Reihe von Forschern, die seit Jahrzehnten Interferenzen zwischen Filmforschung und Literaturwissenschaft zeigen.

Doch ein Bildmedium erzählt anders als ein Wortmedium. Ein Bildzeichen ist leichter mit seinem Bezugsobjekt zu identifizieren als ein Wort: Bild ist semiotisch eine Ikone und dementsprechend unterhält es ein Ähnlichkeitsverhältnis mit seinem Bezugsobjekt: „Die ikonischen Zeichen geben einige Bedingungen der Wahrnehmung

2. - James Monaco, Film verstehen. Kunst, Technik, Sprache, Geschichte und Theorie des Films und der Neuen Medien, Hrsg. Hans-Michael Bock, Reinbek, Rowohlt, 2009, S. 48.

3. - Matías Martinez / Michael Scheffel, Einführung in die Erzähltheorie, München, Beck, 1999, S. 110; dazu auch Kuhn, ,,Minimalbedingung der Narrativität: Es muss mindestens eine Zustandsveränderung in einem gegebenen zeitlichen Intervall dargestellt werden. Der Ausgangszustand vor und der Endzustand nach der Veränderung müssen dabei explizit repräsentiert sein, die Veränderung selbst und ihre Bedingungen nicht." (Markus Kuhn, Filmnarratologie. Ein erzähltheoretisches Analysemodell, Stuttgart, de Gruyter, 2011, S. 21).

4. - Jan Marie Peters, ,,Die Struktur der Filmsprache“, in: Franz-Josef Albersmeier (Hrsg.), Texte zur Theorie des Films, Stuttgart, Reclam, 2003, S. 371-388, hier S. 376.

5. - Ebd. S. 374.

6. - Christian Metz, Langage et cinéma, Paris, Larousse, 1971.

7. - Peter Wollen, Signs and Meaning in the Cinema, Bloomington, Indiana University Press, 1972. 
des Gegenstandes wieder"8. Ferner werden in einer Filmeinstellung Elemente wie Raumtiefe und Farbnuancen immer mitgegeben, was in einem Text nicht von Nöten ist. Der Raum einer Filmeinstellung ist immer voll; er lässt mit Stanzel gesprochen keine „Unbestimmtheitsstellen“9, wie es in Texten gewohnt der Fall ist. So sind Filmeinstellungen in höchstem Grade Abbildungen dessen, was sie darstellen. Die Bilder sind fast die Objekte selbst. Das kann man freilich von Wörtern nicht behaupten. Gleichwohl sind die Einstellungen auf narrativer Ebene in zweierlei Hinsicht komplex: 1. Die Raumfülle kann aufgrund vieler Nebenzeichen die Aussage einer Einstellung problematisieren, da eine Filmeinstellung nicht nur aus dem zentralen Objekt $-\mathrm{z}$. B. einer menschlichen Figur - besteht, sondern auch aus einem Hintergrund mit weiteren Objekten. 2. Die Einstellungen sind in narrative Ketten eingebunden und so gewinnen sie ihre Bedeutung aus ihrem syntagmatischen Gefüge, nicht unbedingt aus einer Ähnlichkeitsrelation mit der direkten Referenz. Es wird am Beispiel zweier Romanverfilmungen - Das Parfum $^{10}$ und Der Vorleser ${ }^{11}$ - zu zeigen sein, dass Filmeinstellungen nicht auf eine eindimensionale Identifikation mit einem außerdiegetischen Objekt reduziert werden können, und dass ihnen mehrfache Funktionen in der syntagmatischen Verkettung filmischer Narration zukommen können.

\section{Referentielle Funktion der Filmeinstellung: Ikone oder Index}

Es ist vorwegzunehmen, dass Bild und Wort beide Abbildungen von Sachen sind. Sprachliches Zeichen und Bildzeichen sind Abstrahierungen von Gegenständen. Das Bild eines Mannes ist genauso wenig ein leiblicher Mann wie das Wort Mann. Peters befindet: „Tatsächlich macht ein Bild die dargestellte Sache nur sichtbar, aber in einer Weise, die stark abweicht von der Sichtbarkeit einer Sache in natura"12. Sehr einleuchtend ist bei Peters, der den Film als Sprache versteht, auch die Differenzierung zwischen Wort und Bild: „Man kann diesen Unterschied auch formulieren, indem man sagt, daß das Wort ein Konzept (eine vom Denken abgeleitete Abstraktion) einer Sache gibt und das Bild ein Perzept (eine von der Wahrnehmung abgeleitete Abstraktion)"13. Das Konzept als eine vom Denken her konstruierte

8. - Umberto Eco, Einführung in die Semiotik, München, Fink, 1994, S. 205.

9. - Franz K. Stanzel, Theorie des Erzählens, Göttingen, UTB, 1995, S. 152 f.

10. - Das Parfum, R.: Tom Tykwer, Deutschland / Frankreich / Spanien / USA, 2006, DVD, Constantin Film, 2007.

11. - Der Vorleser, R.: Stephen Daldry, Deutschland / USA, 2008, DVD, Universum Film, 2009.

12. - Peters, Die Struktur der Filmsprache, a.a.O., S. 372.

13. - Ebd. S. $372 \mathrm{f}$. 
Abstrahierung zu Begreifen mag verwundern. Wörter sind in großem Maße doch arbiträre Zeichen. Nachvollziehbar ist jedoch, das Bild als ein Perzept zu definieren, denn bildliche Zeichen werden visuell wahrgenommen. Somit legt Peters den Grundunterschied zwischen sprachlichem Zeichen und Bildzeichen fest. Bilder sind Perzepte, sie sind visuelle Zeichen, mittels derer wir Gegenstände wahrnehmen. Dieser markante Unterschied problematisiert das Verhältnis zwischen Zeichen und Bezugsobjekt und demzufolge zwischen Zeichen und der erzählten Welt: Bildliche Zeichen ähneln ihren Objekten, sprachliche Zeichen sind aber bis auf onomatopoetische Ausdrücke arbiträr. Bilder im Film sind also Ikonen, Wörter in einem Text eher nicht. So erreicht die Diegese eines Filmes einen höheren Grad der Konkretisierung als Texte. Das stellen mehrere Filmforscher fest.

Die in audiovisuellen Erzählungen dargestellte Welt hat im Vergleich zur Literatur eine weit größere Explizitheit, Anschaulichkeit, Vollständigkeit und Konkretheit. Denn das audiovisuelle Erzählen entspricht - stärker als andere Erzählformen - unserer nichtmedialen, direkten Weltwahrnehmung ${ }^{14}$.

Dieser festen Überzeugung, dass das filmische Bild eine wirklichkeitstaugliche Präsentation liefert, liegt die Annahme zu Grunde, dass ikonische Zeichen notgedrungen eine augenfällige Ähnlichkeit mit ihren Bezugsobjekten tragen, denn „Bilder sind gewiss immer Festlegungen"15. Die Denotate der Zeichen sind im Film etwas strikter definiert als in Texten, weil sie im audiovisuellen Medium Merkmale ihrer Referenten widerspiegeln. Während im sprachlichen Medium der Literatur das Verhältnis des Signifikanten zum Referenzobjekt auf keinerlei Ähnlichkeit beruht, fallen beide im Film aufgrund der Ikonizität quasi zusammen: „Ein Bild hat eine direkte Beziehung zu dem, was es bezeichnet, ein Wort hat das nur selten"16. Demnach wird die Überzeugung fällig, dass ikonische Zeichen im Film der Garant für einen zuverlässigen Zugang zur Filmwelt sind. Kurz: Die Zeichen werden für die Bezugsobjekte selbst gehalten:

In einem mechanischen, ,automatischen“ Abbildungsprozess, so Bazin, überträgt der Gegenstand im photographischen Medium durch einen Effekt der „Wirklichkeitsübertragung“ etwas von seinem Sein auf sein Bild. Das Bild ist demnach nicht nur eine Spur der Realpräsenz

14. - Silke Lahn / Jan Christoph Meister, Einführung in die Erzähltextanalyse, Stuttgart, Metzler, 2008, S. 270

15. - Gottfried Boehm, ,Jenseits der Sprache? Anmerkungen zur Logik der Bilder“, in: Dorothee Kimmich / Rolf G. Renner / Bernd Stiegler (Hrsg.), Texte zur Literaturtheorie der Gegenwart, Stuttgart, Reclam, 2008, S. 476-492, hier S. 490.

16. - Monaco, Film verstehen, a.a.O., S. 169. 
des Dargestellten, sondern eine andere Form der Realpräsenz dieses Dargestellten, eine Form zudem, die nicht für die Wirklichkeit steht, sondern dieser etwas an Sein hinzufügt ${ }^{17}$.

Aus dem ikonischen Verhältnis wird folglich ein eindimensionales Identifikationsverhältnis, ohne Rücksicht auf Ambivalenzen und Mehrdeutigkeiten, aber auch ohne Rücksicht auf das syntaktische Umfeld der (Bild-)Zeichen im Film, das die Zeichenfunktion entscheidend prägt.

Darüber hinaus kommt dem ikonischen Charakter des Filmzeichens ein indexikalischer hinzu. Das Bild ähnelt nicht nur seinem Objekt, sondern es ist auch ein Verweis darauf. Photographisches aber auch filmisches Bild ist insofern ein Index, als es eine Spur ist, die zu einem abgebildeten Objekt hinführt. Es unterhält eine ,real connection with its object" ${ }^{\text {"18 }}$. Ein Index fungiert immer als Verweis auf etwas Anderes, mit dem es in einem kausalen Verhältnis steht: ,they [indices] direct the attention to their objects" 19 . Dem peirceschen Ansatz pflichtet Hediger bei, der das Zeichen im Film ,mit seinem Gegenstand zugleich über eine Beziehung der Ähnlichkeit und eine der physischen Verursachung verbunden" 20 sieht. Das Bild ähnelt nicht nur seinem Objekt, sondern indiziert er auch, d. h. es steht zum Objekt in einer kausalen Beziehung, die die Vergegenwärtigung des Objekts immer betont. Der Fokus liegt in dieser Auffassung des Filmzeichens - ob als Index oder als Ikone auf einer Beziehung zwischen Zeichen und einem Gegenstand in der Wirklichkeit, d. h. einem Gegenstand außerhalb der Filmdiegese; nicht auf der Relation zwischen Zeichen und seinem denotierten Signifikat in der Filmwelt selbst. Gunning argumentiert zum Filmzeichen in diese Richtung: „An indexical argument, as it has been developed, based in the photographic trace, points the image back into the past, to a preexisting object or event whose traces could only testify to its having already been" 21 .

17. - Vinzenz Hediger, ,Illusion und Indexikalität. Filmische Illusion im Zeitalter der postphotographischen Photographie“, in: Deutsche Zeitschrift für Philosophie, Bd. 54 (1), S. 101-110, hier S. 101; dazu auch: Wollen: ,It was the iconic aspect of the sign which Von Sternberg stressed [...] in order to conjure up a world, comprehensible by virtue of resemblances to the natural world" (Wollen, Signs and Meaning in the Cinema, a.a.O., S. 137).

18. - Peirce, Charles Sanders, Collected Papers, Bd. 5., Bd. 1-6 Hrsg. von Charles Harsthorne / Paul Weiss, Bd. 7-8 von Arthur W. Burks, Cambridge / Mass., Harvard University Press, 1931-1958, S. 56.

19. - Ebd., Bd. 2., S. 306.

20. - Hediger, Illusion und Indexikalität, a.a.O., S. 101.

21. - Tom Gunning, ,Moving Away from the Index: Cinema and the Impression of the Reality“, In: Differences. A Journal of Feminist Cultural Studies, Bd. 18 (1), Duke University Press, 2007, S. 29-52, hier S. 47. 
Mit dieser doppelten Funktion des filmischen (Bild-)Zeichens wird der Abstand zum Bezugsobjekt deutlich geringer als in der Literatur. Das betonen nicht nur klassische Abhandlungen zum Film:

Was das filmische Bild von anderen Symboltypen unterscheidet, ist die Tatsache, dass es photographische Qualität exemplifiziert: dass es zu einem Symbolschema von außergewöhnlicher Dichte gehört, bei dem jeder kleinste Unterschied einen Unterschied macht und bei dem der Grad der Abstraktion und der Selektion von wahrnehmbaren Eigenschaften des denotierten Gegenstandes so gering ist, dass Abstraktion und Selektion als solche fast nicht auffallen und also die Zeichenhaftigkeit des Zeichens nur knapp die Schwelle der Wahrnehmung überschreitet ${ }^{22}$.

Dieses Verständnis der Filmzeichen kann der narrativen Strukturierung im Film nicht gerecht werden. Diese Ansätze, die von der Semiotik in der Filmanalyse Gebrauch machen, sehen mehr auf Realreferenzen der Filmzeichen ab, als auf das narrative Interagieren dieser Zeichen. Dabei behält die referentielle Funktion filmischer Zeichen ihre Geltung, wenn auch nur auf einer vordergründigen Ebene.

Tatsächlich - blickt man z. B. auf wenige Einstellungen im Film Der Vorleser, erkennt man sofort die Evokation einer referentiellen Bedeutung, die im Romantext Der Vorleser ${ }^{23}$ nicht intendiert wird. Der Stadtraum findet im Text en passant Erwähnung, nämlich als Randbemerkung zur Fassade des Hauses, in dem Hanna lebt: Das „Haus [war] von den Jahren und vom Rauch der Züge dunkel geworden“ (Vorleser 9) -, im Film wird aber die gesamte Lebenssphäre genauer dargestellt. Die Anfangseinstellungen aus dem Film Der Vorleser vermitteln ein Deutschland der 50er Jahre mit maroden Hausfassaden und einer allgemein tristen Stimmung, in der Michael die Bekanntschaft von Hanna macht. Damit denotieren die ikonischen Zeichen ein Nachkriegsstadtbild, das den Vorstellungen einer deutschen Stadt der 50er Jahre entsprechen könnte. Beglaubigungseffekte sind dabei Verkehrsmittel jener Zeit, z. B. Auto und Bus. So ähneln diese Einstellungen der Wirklichkeit eines Stadtbildes der 50er Jahre. Diese ikonische Funktion bescheinigt dem Film bereits am Anfang ein Echtheitsprädikat und eine anschauliche Fixierung der raumzeitlichen Konstellation. Doch diese Referentialität sagt noch lange nichts über die narrative Strukturierung im Film aus, weil diese erst durch Analyse der syntagmatischen Verkettungen von Zeichen eruiert werden kann. Es wird im Folgenden zu zeigen sein, dass die semiotische Begrifflichkeit

22. - Hediger, Illusion und Indexikalität, a.a.O., S. 108.

23. - Bernhard Schlink, Der Vorleser, Zürich, Diogenes, 1997. 
auf Narrationsebenen in Romanverfilmungen am Beispiel von Das Parfum und Der Vorleser produktiv applizierbar ist.

\section{Indexikalisch-metonymische Funktion der Filmeinstellung}

Der Roman, Der Vorleser, erzählt die Geschichte aus Michaels Sicht, der Hannas Bekanntschaft in einer seiner schweren Stunden macht. Sie verführt ihn, er verliebt sich in sie. Daraus ergibt sich eine Beziehung zwischen beiden, die aus festen Ritualen besteht: ,Vorlesen, duschen, lieben und noch ein bißchen beieinanderliegen - das wurde das Ritual unserer Treffen."(Vorleser 43) Das bildet ungefähr das erste Drittel des Romans, in dem Michael damit beschäftigt ist, seine entdeckte sexuelle Leidenschaft mit Hanna auszuleben. Der Erzählton fällt realistisch aus - wenn auch stark subjektiv eingefärbt. Die Ereignisse erfolgen aus einer kausalen Notwendigkeit aufeinander. Es ist ein ökonomisches Erzählen, das auf kontige Verkettung der Zeichen aufbaut. Die ritualisierten Begegnungen, an die der Text immer wieder erinnert, sind von einem Verhaltenskodex beherrscht, dessen Zeichen beide Beteiligten gut verstehen und respektieren. Der Film thematisiert ebenfalls die Beziehung stark, jedoch mit leichten Deviationen. Als Michael zum zweiten Mal vor dem Haus steht, werden Kohlesäcke ins Haus getragen; erzähltechnisch ein belangloses Objekt, möchte man meinen. Das Bild der Kohlesäcke wiederholt sich in wenigen Einstellungen mehrfach. Unmittelbar darauf wird Michael von Hanna aufgefordert, Kohle nach oben zu bringen. Danach muss er duschen, um dann von Hanna zum Sexualakt verführt zu werden. In diesem Sinn funktionieren die Bilder als Verweise auf kommende Ereignisse. Michael, der an dem mit Kohlesäcken voll beladenen Wagen am Eingang des Hauses vorbeigeht, muss später selbst Kohle schaufeln, was eine Dusche unerlässlich macht, die dann zum unentrinnbaren, aber stark ersehnten Körperkontakt mit Hanna führt. In dieser Hinsicht erfüllen diese Bilder, diese ikonischen Zeichen, im Film in ihrem Zusammenwirken indexikalische Funktionen, da sie kausal aufeinander verweisen. Die Einstellungen gehen ineinander über, sodass Elemente aus den einzelnen Einstellungen ihre Bedeutung erst in der ganzen Sequenz bekommen. Diese Filmtechnik beschreibt Tynjanov folgendermaßen: „Eine Einstellung folgt auf die andere, und jede trägt in sich das bedeutungshafte Zeichen der ihr vorangehenden, ist durch sie in ihrer ganzen Dauer bedeutungsmäßig eingefärbt ${ }^{* 24}$. Narratologisch relevant ist hier nicht, dass das Bild Kohle Ähnlichkeit mit Kohle - die ikonische

24. - Juri N. Tynjanov, „Über die Grundlagen des Films“, in: Franz-Josef Albersmeier (Hrsg.), Texte zur Theorie des Films, Stuttgart, Reclam, 2003, S. 138-171, hier S. 143. 
Funktion also - in Wirklichkeit hat, sondern die Relation des Zeichens Kohle mit den darauf folgenden Einstellungen in der ganzen Sequenz. Im Verlauf des Textes besteht diese Formel weiter, jedoch mit einer Substitution: Der Arbeitsaufwand, Kohle schaufeln, wird durch Lesen substituiert; Michael muss keine grobe Arbeit verrichten, damit er mit einer Dusche und einem Liebesakt entlohnt wird, sondern sich intellektuell betätigen. An den weiteren Abschnitten des Rituals ändert sich nichts: Lesen - Duschen - Lieben. Der Film gibt diese drei Abschnitte in Bildeinstellungen wieder, in denen der eine Vorgang den nächsten indiziert. In diesem Sinne kann das Lesen als Index für die Dusche, die Dusche ebenfalls als Index für den Liebesakt verstanden werden. So erfüllen die ikonischen Zeichen in der Filmdiegese auf narrativer Ebene eine indexikalische Funktion.

Der indexikalischen Funktion, die für eine Verkettung der Ereignisse sorgt, ist eine metonymische Funktion inhärent. Michael liest Hanna aus Schulliteratur vor; z. B. Emilia Galotti und Die Odyssee. Dass Michael Hanna aus diesen Büchern vorliest, impliziert ihre Einweihung ins Schulwesen und ihre langsame Befreiung vom Analphabetentum. Mit Michael kommt folglich ein Stück Schule in Hannas Wohnung. Die Einstellungen, die die Bücher zeigen, exemplifizieren auf metonymische Art Hannas Annäherung an die Bildung. Diese ikonischen Zeichen, die Bücher, die teilweise die Bildoberfläche füllen, sind also erzähltechnisch als Pars pro Toto zu verstehen: Die Schulliteratur steht für Schule und demzufolge für Bildung, an der Hanna teilhaben will. Die primären Referenzen sind in der Konstellation von zweitrangiger Bedeutung; vielmehr signalisiert das Vorlesen aus diesen Büchern einen Lernprozess, der bei Hanna schon früher angefangen hat- als KZ-Aufseherin ließ sie sich dort von Gefangenen aus Büchern vorlesen - und sich später im Gefängnis fortsetzen wird. Somit erfüllen die Filmeinstellungen in ihrer Verkettung eine indexikalisch-metonymische Funktion, die den Fortgang der Diegese begünstigen.

Ein Beispiel des Bild-Erzählens aus dem Film Das Parfum soll diese narrative Funktion ikonischer Zeichen noch mehr verdeutlichen. Der Roman erzählt die Geschichte des Jean-Baptiste Grenouille, der über einen außergewöhnlichen Geruchssinn verfügt, selbst aber keinen Geruch hat. Er sammelt Gerüche junger Frauen, die er tötet und deren Gerüche destilliert, bis er zum Schluss daraus sein Parfum schafft. Seine erste Mordtat erfolgt nach einer Begegnung mit einem Mädchen, das einen Mirabellenkorb in der Hand trägt. Der Romantext beschreibt die Begegnung mit dem Mädchen als eine Begegnung mit dem Geruch: Grenouille dachte, ,er habe in seinem Leben noch nie etwas so Schönes gesehen, wie dieses Mädchen. [...] Er meinte natürlich, er habe noch nie so etwas Schönes gerochen.“ (Das Parfum 54). Es ist also eine explizite Konzentration auf den Geruch, dem der Text mit allerlei Schilderungen 
der Geruchsnoten beizukommen, intendiert. Der Romantext verfolgt mehr die Geruchswahrnehmung, z. B. mittels Signifikantenhäufungen. Das Mädchen selbst wird nicht intensiv beschrieben wie die Gerüche, die es ausscheidet:

Ihr Schweiß duftete so frisch wie Meerwind, der Talg ihrer Haare so süß wie Nußöl, ihr Geschlecht wie ein Bouquet von Wasserlilien, die Haut wie Aprikosenblüte..., und die Verbindungen all dieser Komponenten ergab ein Parfum so reich, so balanciert, so zauberhaft, daß alles, was Grenouille bisher an Parfums gerochen, alles, was er selbst in seinem Innern an Geruchsgebäuden spielerisch erschaffen hatte, mit einem Mal zu schierer Sinnlosigkeit verkam ${ }^{25}$.

Der Film aber vollzieht großformatige Darstellungen des Mädchens und rückt Gesicht, Haare, Brüste usw. in den Vordergrund. Die Verfolgung des Geruchs, auf den Geruchszeichen hinweisen, wird zu einer Verfolgung des Mädchens. So taucht das Mädchen in der Filmsequenz schon früher auf als im Textabschnitt und es kommt zu einem ersten Kontakt, doch das Mädchen rennt weg. Der unerbittliche Grenouille folgt ihr jedoch, bis er all ihre Gerüche in sich aufnimmt. Die Filmeinstellungen versuchen dem Umstand Rechnung zu tragen, textuell dargestellte Gerüche und Geruchswahrnehmung bildlich zu präsentieren. So kulminiert im Film die Geruchsekstase, in der sich Grenouille nun befindet, im Bild des Mädchens, respektive in der Ikone des Mädchens, die aber in der Engführung mit Grenouilles Riechaktivitäten in der Filmmontage zum Zeichen ihres Geruchs wird: Je stärker das Mädchen ins zentrale Geschehen rückt und sein Gesicht und Körper die Bildfläche füllen, desto intensiver die Exposition der Geruchswahrnehmung. Das Mädchen mit dem Mirabellenkorb ist in diesem Sinne eine Metonymie; es geht in dieser Sequenz nicht um die direkte Referenz rothaariges Mädchen, sondern um seinen Geruch. Das Mädchen indiziert den Geruch: Es ist mit Eco gesprochen ein Aufmerksamkeitsvektor, der Grenouilles Riechsinn lenkt. „Indizes [sind] Aufmerksamkeitsvektoren" 26 . Ferner - und zwar auf der breiten Ebene der Filmdiegese - präfigurieren die großformatigen Aufnahmen von Grenouilles Nase intensive Geruchswahrnehmungen, die in schlüssigen Stellen auf junge Mädchen schließen, die er erreicht und tötet, um von ihren Körperdüfte Besitz zu ergreifen. Nach diesem Muster erfolgt sein letzter Mord:

25. - Patrick Süskind, Das Parfum. Die Geschichte eines Mörders, Zürich, Diogenes, 1994, S. 54 f.

26. - Umberto Eco, Zeichen. Einfiihrung in einen Begriff und seine Geschichte, Frankfurt a.M., Suhrkamp, 1977, S. 157. 
Grenouilles Nase wird gezoomt (Geruchswahrnehmung), darauf folgt die Bildeinstellung des jungen Mädchens, dann die nächsten Schritte: Mord, Enthaarung, Sammlung der Körpergerüche und als letzte Etappe die Geruchsdestillierung. Dieses Schema bildet im Parfum einen stabilen Handlungsstrang, in dem ikonische Zeichen wie im Vorleser in indexikalisch-metonymische Funktionen umgemünzt werden. Natürlich sind das keine genuinen Indexe, wie sie Semiotiker verstehen. Doch ein Index ist immer eine Spur, ein Verweis, ein „Anzeichen“27, und in diesem Sinn verwandeln sich in diesen Romanverfilmungen einige Bildzeichen - eigentliche Ikonen - in Indexe, durch eine Form der Verkettung. Jedes der obigen vier Bilder trägt einen Verweis, der wie ein Vektor auf das nächste Bild zeigt. So entsteht im narrativen Kontext des Films eine kausale Verknüpfung der Einstellungen.

Es ist also festzustellen, dass Filmeinstellungen, Bildzeichen, eine indexikalisch-metonymische Funktion zukommt, die die Narration in der Filmdiegese durch kausale Verweisstrukturen garantiert. Die Handlung treiben ikonische Zeichen voran, nicht durch ihre primären Referenzen sondern durch ihre Einbindung in syntagmatische Verkettungen. Doch die Spannweite der ikonischen Zeichen im Film erschöpft sich nicht in der indexikalisch-metonymischen Funktion. Es geht auch um Symbolgehalte, die auf konnotativer Ebene in Erscheinung treten.

\section{Symbolische Funktion der Ikonen}

Konnotationen im Film liegen vor, ,,wenn es erst einmal in seiner genauen syntagmatischen Position innerhalb der Rede, die der gesamte Film bildet, lokalisiert ist, gelingt es einem visuellen oder auditiven Motiv - oder auch einer Anordnung von visuellen oder auditiven Motivaten -, mehr zu sein, als es ist, und eine Sinnerweiterung zu erhalten"28. Diese Definition von Metz hat bis heute noch Geltung. Dem beipflichtend definiert Thompson die Konnotation wie folgt: „Konnotationen können als implizite Bedeutungen (implicit meanings) auftauchen, auf die das Werk verweist" 29.

Filmeinstellungen bestehen meistens nicht aus fixen Porträtierungen der Figuren oder aus einzelnen Gegenständen vor leerem Hintergrund. Weitere Elemente wie Raumtiefe und Kostümierung sind Faktoren

27. - Vgl. Uwe Wirth, ,Spuren am Rande. Zwischen genuiner und degenerierter Indexikalität" “, in: Heike Gfrereis / Marcel Lepper (Hrsg.), deixis - vom Denken mit dem Zeigefinger, Göttingen, Wallstein Verlag, 2007, S. 181-195, hier S. 184.

28. - Metz, Probleme der Denotation im Spielfilm, a.a.O., S. 323.

29. - Kristin Thompson, „Neoformalistische Filmanalyse“, in: Franz-Josef Albersmeier (Hrsg.), Texte zur Theorie des Films, Stuttgart, Reclam, 2003, S. 427-464, hier S. 433. 
von erheblicher Eminenz, denn im Film muss die Raumtiefe gefüllt werden. Damit weist der Film ein Charakteristikum auf, das in Texten nicht auftritt. Solche Elemente können Einstellungen und Sequenzen mit einem Bedeutungsmehrwert aufladen, den im Text nicht-visuelle Zeichen artikulieren. Ferner kann der Mehrwert mancher Einstellungen mit dem anderer Einstellungen korrespondieren. So kann man von einer weiteren Bedeutungsebene, einer symbolischen Bedeutungsebene, sprechen.

Im Film Das Parfum ist eine sehr kurze Einstellung zu verzeichnen, in der ein blauer Stoff in den Vordergrund tritt. Diese Einstellung ist Teil einer langen Sequenz, in der Grenouille durch die Straßen von Paris umher läuft und alle Gerüche in sich hinein saugt.

Wählerisch ging er nicht vor. Zwischen dem, was landläufig als guter oder schlechter Geruch bezeichnet wurde, unterschied er nicht, noch nicht. Er war gierig. Das Ziel seiner Jagden bestand darin, schlichtweg alles zu besitzen, was die Welt an Gerüchen zu bieten hatte (Das Parfum 48),

so der Roman. Ein Hinweis auf einen blauen Stoff fehlt jedoch, wie bei der ersten Begegnung mit dem Mädchen in Grasse, die rein olfaktorisch ausfällt: „Grenouille sah dieses Mädchen in seiner olfaktorischen Vorstellung wie auf einem Bilde vor sich“ (Das Parfum 216). Der Film macht aber aus dieser Geruchsbegegnung eine visuelle Begegnung am Grabmal der Mutter des Mädchens im großen Garten der Familie. Das Mädchen trägt ein blaues Kleid. Diese blaue Kostümierung wird am Ende des Textes nach vollbrachtem Werk, d. h. nachdem Grenouille obiges Mädchen umgebracht und dessen Geruch seinem Parfum hinzugefügt hat, in Erscheinung treten. Nun trägt Grenouille einen blauen Anzug. Diese blaue Motivik im Film konstruiert ein Setting, das vom Text abweicht. Das Blaue als Stoffmotiv am Anfang und fast flüchtig wahrnehmbar, dann als Kleid des Mädchens, dessen Geruch Grenouille im Text zum höchsten Duftprinzip erhöht, und letztendlich als Grenouilles Anzug selbst, unterstützt im Film eine andere Bedeutungsebene. Das Kleid des Mädchens ist mehr als ein Kleidungsstück. Es inkorporiert eine Sehnsuchts- und Liebesfigur: Es ist Abend, Grenouille schleicht sich in den Garten. Das Mädchen kommt in einem blauen Kleid und trägt eine weiße Rose zum Grab der Mutter: Sowohl raumzeitliche Disposition - Abend im Park - als auch Farbsymbolik inszenieren eine unstillbare Sehnsucht nach einem Liebesobjekt, das in greifbarer Nähe ist, jedoch nicht erreichbar. Nicht nur die blaue Farbe demonstriert das, sondern auch die weiße Blume, die natürlich die Trauer des Abschieds von der geliebten Mutter symbolisiert, aber auch Grenouilles Sehnsucht ins Ewige dehnt. Für ihn ist, die Liebe zu erreichen, genauso wenig zu 
realisieren, wie Tote ins Leben zurückzurufen. Diese Ikonen, das blaue Kleid und die weiße Rose, sind Zeichen romantischer Provenienz. Inkorporiert das blaue Kleid Grenouilles Sehnsucht - Blau ist ja dezidiert die Farbe der Sehnsucht in der romantischen Literatur -, so versinnbildlicht die weiße Rose die Unerreichbarkeit der ersehnten Geliebte. Diese Symbolebene der ikonischen Zeichen im Film stützt ein Paradigma der Sehnsucht, das im Text in dieser Form nicht dargeboten wird. Diese zentrale Sequenz im Film wird an später Stelle wieder aufgegriffen: Der Triumph im blauen Anzug vor den Grasser Massen ist konnotativ gesprochen der Triumph, in die Haut des geliebten Mädchens schlüpfen zu können, nachdem Grenouille sein Werk, sein Parfum, vollbracht hat. Diese Vollendung treibt Grenouille jedoch Tränen in die Augen, die ihn sehr weit zurückwerfen, zu seinem ersten Mord, zum ersten geliebten Mädchen mit dem Mirabellenkorb: Der Film vollzieht eine Bildmontage zwischen Grenouille im blauen Gewand auf der vom Richtplatz zum Siegespult verwandelten Stelle und dem phantasmatischen Liebesakt mit dem Mädchen, das in ihm erste Sehnsüchte erweckt hat. Somit schließt sich der symbolische Kreis einer Liebesgeschichte von unstillbarer Sehnsucht. Die Responsionen zwischen diesen ikonischen Zeichen, die allesamt eine Sehnsuchtssymbolik beschwören, transponieren die gesamte Geschichte eines mörderischen Genie-Parfümeurs in eine rührende Geschichte der Sehnsucht.

Mithin wird deutlich, dass die Einstellungen im Film über ihre indexikalische Funktion in der Filmdiegese als Verkettung von Ereignissen, die Grenouille zur Herstellung eines Parfums aus den Gerüchen junger Mädchen verhelfen, hinausgehen und eine symbolische Ebene schaffen, die Grenouilles Geschichte als Parfümeur in eine Liebesgeschichte umcodiert ${ }^{30}$. Die Ikonen im Film arbeiten in diesem Sinne auf zwei Ebenen: auf einer indexikalisch-metonymischen Ebene, die den Fortgang des Filmgeschehens stützt und einer symbolischen Ebene.

Im Film Der Vorleser ist Ähnliches festzustellen. Hanna war bei der SS als Aufseherin in einem Lager. Frauen waren in eine Kirche eingesperrt, die von einer Bombe getroffen wird und Feuer fängt. Die Mehrzahl der eingesperrten Frauen stirbt beim Brand. Hanna und die anderen Aufseherinnen werden beschuldigt, die Tür der Kirche geschlossen zu halten und so die Frauen nicht vor dem Brand zu retten. Während der Text Hanna anhand falscher Kausalität entlastet - der Grund für ihre Taten bzw. Untat im Nationalsozialismus sei ihr Analphabetentum, setzt die Ikonik des Filmes auf eine andere Technik, die Hanna - weil

30. - Vgl. Wolfgang Delseit und Hannes Fricke, „Das Parfum (Patrick Süskind - Tom Tykwer). Positiver Held und unbegreifliches Genie: Über die Schwierigkeiten, der (eigenen) Filmsprache zu vertrauen“, in: Anne Bohnenkamp (Hrsg.), Literaturverfilmungen, Reclam, Stuttgart, 2012, S. 351-368, hier S. 356 f. 
zur Alleinschuldigen erklärt wird, Analphabetin ist und dies aus Scham nicht verraten will - von der Rolle der Mittäterschaft subtil in die Rolle des Opfers schlüpfen lässt. Der Film zeigt Einstellungen einer ausgelieferten Hanna im Gewand eines den Tränen nahen Opfers. Allein kämpft sie im Prozess gegen die Justiz und die anderen Aufseherinnen, die im Film anders als Hanna vom Justizpersonal abgeschirmt, gemütlich dasitzen und nicht einzeln verhört werden, dabei richtig grimmig und böse wirken und gemeinsam gegen Hanna aussagen. Mit diesen Bildeinstellungen übertrifft der Film den Text. Die Möglichkeiten, die den ikonischen Zeichen im Film zukommen, erlauben eine neue Argumentationsschiene. Die Sequenz der Gerichtsverhandlung zeigt auf der denotativen Ebene eine Hanna Schmitz, die aufgrund eines falschen Schuldbekenntnisses und schlechten Verhaltens zu 19 Jahren Haft verurteilt wird. Doch die betonte räumliche Isolierung Hannas im Gerichtssaal, die in mehrfachen Einstellungen hervorgehoben wird und ihre ins Gesicht geschriebene ausdrückliche Hilflosigkeit erzeugen einen Effekt der Rührung. Hanna wird bemitleidenswert.

In der geschickten Emotionalisierung des Zuschauers wird Hanna in dem Augenblick zur Heldin, als sie die Verantwortung, die ihr von den Mitangeklagten untergeschoben wird, auf sich nimmt. Die schöne, schüchterne, freundliche Hanna wird im Zeugenstand zum Opfer, das auf das Konto der grimmigen, verhärmten, alten Aufseherinnen auf der Anklagebank geht ${ }^{31}$.

Die ikonischen Zeichen implizieren in diesem Sinne ein Opfer. Die ganze Gerichtsverhandlung, die auf den ersten Blick im Film und Text wohl gegen Hanna verläuft, verleiht ihr tragische Größe: Hanna sitzt im Zentrum des Gerichtssaals und trägt auf ihren Schultern die Schuld aller. Somit wird Hanna Schmitz, die Aufseherin im Lager, die Frauen und Kinder in einer Kirche verbrennen ließ, gegen das Bild einer Hanna von tragischer Größe ausgetauscht. Ihre Ikone im Gerichtssaal konnotiert ein tragisches Opfer.

Dieses Symbol des tragischen Opfers wird nicht nur in dieser Sequenz proklamiert, sondern im ganzen Film angedeutet. Sehr früh, noch bevor Michael von Hannas Vergangenheit bei der SS erfährt und auf einem Ausflug, den sie gemeinsam unternehmen, gehen beide in eine Kirche; der Chorgesang treibt Hanna Tränen in die Augen. Ihre groben Umgangsformen treten dabei zugunsten einer weicheren Figur zurück. Jedoch wird die Einstellung in der Kirche im Hinblick auf die späten Enthüllungen ihrer Vergangenheit insofern evident, als sie Hannas Reue und Schuldbewusstsein vorwegnimmt, Jahre bevor der

31. - Michael André, Hanna, mon Amour, in: http://www.freitag .de/autoren/derfreitag/hanna-mon-amour (26.02.2009). 
Prozess beginnt. Die Einstellung in der Kirche konnotiert eine früh ansetzende Auseinandersetzung mit der eigenen Schuld.

Nach der Gerichtsverhandlung besucht Michael das Konzentrationslager Struthof im Elsass: „Das Lager war geschlossen“ (148) heißt es im Text. Michael, der versucht, den „Zivilisationsbruch“"32, zu verstehen, steht vor der Unmöglichkeit der Erinnerung: Die Vorstellung, wie es darin war, ist nicht zu vergegenwärtigen (149): „Aber die fremde Welt der Konzentrationslager war mir darum nicht nähergerückt.“ (152) Die direkte Botschaft dieser Szene ist die aus Michaels Sicht dargestellte Unmöglichkeit, die Vergangenheit zu verstehen, d. h. die Vergangenheit sichtbar zu machen.

Doch die kinematographische Inszenierung der Sequenz liefert eine eindringliche Verfremdung, nahezu ständig in grauem Farbton: Drahtgitterzaun, Kästen voller Schuhe, Schlaflager, Krematorium, alles in schwerer Düsternis. Dieses graue Bündel von Zeichen - auch in diesem Film springt Farbikonik ins Auge - denotiert unmissverständlich, wie für Michael der Nationalsozialismus ein fremder mit Stacheldraht umzäunter Raum geworden ist, der eher geheimnisvoll wirkt als Erklärungen anbietet, konnotiert gleichwohl eine große unüberbrückbare raumzeitliche Distanz zu einer Zeit, in der Hanna schuldig war. Darin steckt eine Volte des Films: Die heutige Hanna vor dem Gericht trägt kaum Spuren jener Zeit, womöglich weil sie mit der Aufarbeitung selbständig schon lange begonnen hat, wie die Einstellung in der Kirche einräumt. Die Grausamkeiten des Nationalsozialismus stehen jedoch den anderen Aufseherinnen ins Gesicht geschrieben. Makellos sitzt aber Hanna vor dem Richter und sagt aus. Obwohl sie dabei aufgrund fehlerhafter Beweisaufnahme vom Gericht schuldig gesprochen wird, wird sie sowohl im Romantext als auch im Film zur Sympathieträgerin verklärt. Damit spinnt sich auch in diesem Film eine narrative Ebene, die die primäre Referenzebene überwindet und die Diegese des Films von einem Nazi-Prozess in nahezu eine Verurteilung einer Unschuldigen verwandelt; diese Intention ist implizit und wird von ikonischen Zeichen getragen, die auf symbolischer Ebene interagieren.

Die Analyse zeigt, dass die Romanverfilmungen keine simple Umwandlung von Textnarrationen in Filmnarrationen sind, sondern dass die Bilder als ikonische Zeichen, die ebenfalls von der Filmtheorie des Öfteren auch als Indexe verstanden werden, auf narrativer Ebene eine doppelte Funktion erringen: 1. Die Einstellungen als Ikonen konstruieren insofern narrative Settings als sie aufeinander verweisen, d. h. eine Einstellung indiziert die nächste und jede Einstellung verar-

32. - Klaus Köhler, Alles in Butter. Wie Walter Kempowski, Bernhard Schlink und Martin Walser den Zivilisationsbruch unter den Teppich kehren, Würzburg, Königshausen \& Neumann, 2009, S. 253. 
beitet weiter den Inhalt der vorhergegangenen. Bei diesem Interagieren ikonischer Zeichen ist nicht die primäre Referenz auf ein Bezugsobjekt relevant, sondern das kausale Umfeld. Die Abhandlung definiert diese Verkettung von Einstellungen als indexikalisch-metonymische Funktion im Film, die aus Ikonenkombination hervorgeht. 2. Ikonen stuitzen die Herausbildung von Symbolebenen, die implizite Bedeutungsinhalte fördern. Natürlich, nicht jede Einstellung, nicht jede Ikone, hat eine symbolhafte Dimension, sondern bestimmte, die Responsionen mit anderen Zeichen bilden und eine gefestigte mittelbare Bedeutungsebene kreieren. Das ist alles der Verdienst der Ikonen im Film, weil sie gerade in einer filmischen Narration nicht die bildliche isolierte Identifikation mit dem, was sie abbilden, sind, sondern von ihrem syntaktischen Umfeld abhängen, und zwar auf indexikalisch-metonymischer, aber auch auf konnotativer Ebene. Ikonische Zeichen bilden im Film Syntagmen auf denotativer und konnotativer Ebene. 
